\title{
Cost effectiveness of the implantable cardioverter defibrillator: a preliminary analysis
}

Department of Clinical Epidemiology and Biostatistics, McMaster University, Hamilton, Ontario Canada B J O'Brien

Health Economics Research Group, Brunel University, Uxbridge

M J Buxton

Health Policy Unit, London School of Hygiene and Tropical Medicine, London J A Rushby

Correspondence to Dr B J O'Brien, Hamilton Civic Hospitals Research Centre, Henderson Genera Division, 711 Concession Street, Hamilton, Ontario, L8V 1C3, Canada.

Accepted for publication 28 November 1991

\begin{abstract}
Background-An implantable cardioverter defibrillator (ICD) may be effective in reducing the risk of sudden cardiac death. The high cost of ICD treatment, however, compared with alternatives raises the question of whether this new technology is an efficient use of scarce health care resources.
\end{abstract}

Objective-To estimate the incremental cost effectiveness of the implantable cardioverter defibrillator compared with drug treatment with amiodarone in the management of patients at high risk of sudden cardiac death.

Design-A cost effectiveness model was constructed from data already published and other secondary sources. Differences in patient survival were calculated from life tables for comparable ICD and amiodarone patient series. Costs were based on typical patient management protocols derived from current United Kingdom practice and interviews with physicians.

Main outcome measures-Cost effectiveness of ICD treatment was computed over 20 years; all future costs and effects were discounted at $6 \%$ per year.

Results-Estimated life expectancy was 11.1 and 6.7 years with ICD and amiodarone respectively; the discounted 20 year difference lies in the range 1.7 to 3.7 years. Discounted 20 year treatment costs were $£ 28400$ for the ICD and $£ 2300$ for amiodarone. Cost effectiveness of ICD treatment lies in the range of $£ 15400$ to $\$ 8200$ per life-year gained.

Conclusions-Cost effectiveness of ICD treatment is similar to some existing cardiac programmes funded under the NHS but uncertainty exists due to limitations of the data. Costs of ICD treatment may fall in the future as the life of the device increases and less invasive implantation methods are needed. The effectivess of ICD compared with amiodarone is currently being studied by a randomised controlled trial.

\section{(Br Heart J 1992;68:241-5)}

The implantable cardioverter defibrillator (ICD) was first implanted into a human patient in $1980^{1}$ and since then it has gained wider use in the management of patients at risk of sudden cardiac death due to ventricular tachycardia (VT) or ventricular fibrillation (VF). In 1990 there were estimated to be over 4000 new implants in the United States whereas the total in the United Kingdom in January 1990 was reported to be 49 devices implanted into 40 patients. ${ }^{3}$

The ICD is an electronic device designed to monitor the heartbeat, recognise VT or VF, and deliver an electric shock (up to 40 joules) to stop the life threatening arrhythmia. Within the generic classification of ICD there are various types, but all consist of a battery powered pulse generator that is implanted in a subcutaneous abdominal pocket and capable of delivering shocks to the heart through two or three electrodes that may be inside or outside the heart.

Use of an ICD is generally considered in patients at high risk of VT, VF and sudden cardiac death, specifically in survivors of sudden cardiac death where electrophysiological study, treadmill, and holter study have failed to identify an effective antiarrhythmic drug. In such patients the main therapeutic alternatives are (a) to implant an ICD that will terminate arrhythmias when they occur; (b) surgical management such as endocardial resection where an attempt is made to remedy the cause of the arrhythmias; (c) empirical drug treatment with amiodarone where the goal is to prevent arrhythmias occurring.

In this study we compare the costs and effects of treatment with ICD versus the alternative amiodarone. Although these are not the only comparisons that could be made they are relevant comparisons that are prevalent in studies examining ICD efficacy. ${ }^{45}$ The relevance of this comparison is also supported by the existence of a large continuing Canadian randomised controlled trial of amiodarone versus ICD treatment. ${ }^{6}$

As well as the improved efficacy of ICD management is the expectation of higher costs, with the device alone costing about f11000. Economic evaluation is therefore a necessary and important feature of any overall assessment of this new medical technology: to what extent is ICD treatment a cost-effective addition to the management of patients at risk of sudden death and how does it compare with other cardiac procedures that are competing 
for scarce health care resources? This study is a preliminary assessment of the economics of ICD treatment in the United Kingdom in which we use the limited published data available to estimate cost effectiveness under various possible outcomes of ICD technology in the near future.

\section{Patients and methods \\ MORTALITY}

No randomised controlled trial of amiodarone versus ICD treatment for patients at high risk of VT or VF has yet been reported. Among the assessments of ICD efficacy, two studies have compared the device with amiodarone. Fogoros et al analysed a series of 78 consecutive patients who were eligible for ICD, a third of whom did not receive the device because of temporary unavailability. ${ }^{4}$ Although this natural experiment was suggestive of a considerable mortality benefit from ICD compared with amiodarone the results are atypical because the sudden death rate of $31 \%$ in the 29 patients taking amiodarone was three times higher than the $9 \%$ sudden death rate reported in the largest published series of 462 patients taking this drug. ${ }^{7}$ Also a case control study, which is reported in abstract, of ICD versus drug treatment is indicative of ICD mortality benefit, but this is of limited value for the present comparison because only $43 \%$ of control patients received amiodarone.

In the absence of reliable data on relative efficacy from a clinical experiment of amiodarone versus ICD, outcomes for our study of cost effectiveness are based upon the largest published patient series for each treatment. Data on survival after ICD implantation were taken from the follow up study of 270 patients by Winkle $e t a l^{8}$ in the United States. In this consecutive patient series the mean age was 58 (range 12 to 77 ) and $80 \%$ were men. There was a history of sustained ventricular tachycardia or fibrillation in $96 \%$ of patients and $80 \%$ had experienced one or more earlier cardiac arrests. Two hundred and sixty nine patients $(96 \%)$ had experienced at least one failure of antiarrhythmic drug either clinically or at an electrophysiological study, and the average number of drug failures was 3.4 for the entire group.

Data on survival with amiodarone treatment were taken from the follow up study of 462 patients by Herre et al. ${ }^{7}$ The mean age in this consecutive patient series was 61 and $80 \%$ were men. Patients were eligible for amiodarone treatment if they had documented sustained ventricular tachycardia or had experienced cardiac arrest. Before initiation of amiodarone treatment, all patients had experienced failure of at least one antiarrhythmic drug either clinically or at electrophysiological study, and on average there were 2.4 drug failures. These characteristics suggest that many of the patients in the amiodarone series would have been eligible for ICD implantation.

\section{LIFE EXPECTANCY}

An assumption of our analysis was that differential patient life expectancy between the treat- ment groups would be largely a function of differences in rates of sudden cardiac death, because neither treatment is presumed to reduce deaths other than sudden cardiac deaths. Therefore, compared with the amiodarone series, the lower rate of other deaths reported in the ICD series suggests that one or more unknown prognostic factors are unequally distributed between the groups. ${ }^{7} \mathrm{We}$ have attempted to compensate for this bias by calculating life expectancies with an adjusted all cause mortality where both treatment groups were assumed to have the same nonsudden death rate, this being arbitrarily set to the mean of the non-sudden death rates for the two groups combined for each follow up time. Also for comparison, all life expectancy and cost effectiveness estimates are made with the unadjusted all cause mortality from the published series.

Patient life expectancy is calculated from the adjusted and unadjusted five year mortality series for both treatments by the declining exponential approximation to life expectancy (DEALE) method proposed by Beck et al. ${ }^{9}$ This method permits declining exponential survival models to be estimated for each five year mortality series, thus enabling forward projections to calculate life expectancies. The DEALE method has been used in previous cardiac studies and the main assumption of the method is that life expectancy approximates to the reciprocal of the annual mortality. ${ }^{10}$

\section{costs}

For items such as the ICD device and prescription drugs, costs can be based on market prices, but limited data are available on the costs of non-marketed health care items such as ICD implantation surgery; NHS accounting systems do not generate information in a form that can easily be used for the routine costing of specific interventions. Ideally, a detailed prospective costing study of antiarrhythmic treatment would be undertaken, as was done with heart transplantation in the United Kingdom. ${ }^{11}$ But the reliability of this method applied to ICD treatment in the United Kingdom might be suspect for two reasons: the small numbers of patients who have received ICDs (49 devices in 40 patients by January $1990^{3}$ ) and the rapid changes in ICD technology.

The approach used by us is to combine costs and published data with information from physician interviews to build protocols of resources used for typical ICD and amiodarone patient management in the United Kingdom. Table 1 summarises these protocols. They were costed by two main sources of data: $(a)$ national published data on hospital costs and outpatient visits; $(b)$ published data from a recent detailed study of costs of various types of cardiac surgery. ${ }^{12}$

The most common method of ICD implantation, left lateral thoracotomy, ${ }^{38}$ was judged to be between a planned coronary artery bypass graft and an adult closed heart procedure in terms of resource use. It was costed at $£ 3000$ exclusive of device generator $(£ 9500)$ plus leads and patches $(£ 1200)$. Replacement of the 
Table 1 Typical patient management protocols for ICD and amiodarone treatment

\begin{tabular}{|c|c|c|}
\hline & ICD treatment & Amiodarone treatment \\
\hline $\begin{array}{l}\text { Initial treatment } \\
\text { In hospital } \\
\text { Main materials } \\
\text { Main procedures }\end{array}$ & $\begin{array}{l}1 \text { day intensive care unit, } 7-11 \text { days on ward } \\
\text { ICD generator, leads, patches } \\
\text { Surgical implantation (lateral thoracotomy); EP study to } \\
\text { test device after implantation }\end{array}$ & $\begin{array}{l}1 \text { week on ward } \\
\text { Amiodarone } \\
\text { Further electrophysiological evaluation }\end{array}$ \\
\hline $\begin{array}{l}\text { Routine follow up } \\
\text { Hospital visits }\end{array}$ & $\begin{array}{l}\text { Outpatient visit every } 3 \text { months; } 1 \text { inpatient day every } 4 \\
\text { years to replace generator }\end{array}$ & Outpatient visits every 3 months \\
\hline $\begin{array}{l}\text { Main materials } \\
\text { Main procedures }\end{array}$ & $\begin{array}{l}\text { Device generator replaced every } 4 \text { years } \\
\text { Device interrogation and battery checking (outpatient); } \\
\text { device replacement under general anaesthetic (inpatient) }\end{array}$ & $\begin{array}{l}\text { Amiodarone (oral) daily ( } 400 \mathrm{mg} \text { ) } \\
\text { Tests to monitor for drug toxicity }\end{array}$ \\
\hline
\end{tabular}

ICD generator, on average every four years, was costed as equivalent to a pacemaker replacement $(£ 750)^{12}$ and added to this are the costs of routine three-monthly follow ups for device and battery checks.

Consistent with the patient series of Winkle et $a l^{8}$ it was assumed that $23 \%$ of patients with an ICD would also be taking amiodarone. Patients receiving only amiodarone were assumed to spend one week in hospital for a loading dose of the drug and further EP evaluation $(£ 1050)$ then an oral daily dose of $400 \mathrm{mg}$ in each subsequent year $(£ 223$ per year) with outpatient visits to monitor for toxicity every three months ( $£ 80$ per year).

Given limitations of the data the cost analysis contains several simplifying assumptions. For example, no explicit additional costs are incorporated into the amiodarone treatment group for management of recurrence of arrhythmia, drug intolerance, or discontinuation of the drug. These costs will be at least partly offset by additional costs in the ICD group for the management of complications such as infections and false shocks.

\section{COST EFFECTIVENESS}

The purpose of the analysis of cost effectiveness is to determine at what extra cost the extra lifeyears of patient benefit result from ICD implantation. All analyses are relative to the alternative of amiodarone treatment, and therefore the cost effectiveness of ICD treatment is the ratio of the net cost of ICD treatment (ICD total costs minus amiodarone total costs) to the net benefits measured as life-years gained (or lost).

Given that costs and benefits occur at different times in the future it is necessary to discount the future trends into present values. The need to discount in economic appraisal merely reflects the fact that individuals have a positive rate of time preference and prefer benefits sooner and costs later. ${ }^{13}$ The discount rate used is $6 \%$ per annum as recommended by $\mathrm{Her}$ Majesty's Treasury for public sector appraisals.

Sensitivity analysis was performed on several variables where limited data were available and uncertainty exists. The purpose of sensitivity analysis is to explore the extent to which the estimated cost effectiveness results stand up to alternative estimates and assumptions conerning key variables. We conducted a two way analysis of sensitivity: ( $a$ ) by mortality series, adjusted versus unadjusted; $(b)$ by alternative assumptions about future treatment and costs. Whereas some alternative assumptions are based on present trends in management (for example reduced follow up times and increased generator life) others, such as the possible reduction in the price of the device due to increased market competition, are more speculative.

\section{Results}

Table 2 shows the five year mortality data for ICD and amiodarone treatment. At five years the sudden and non-sudden death rates for ICD treatment were $4.4 \%$ and $21.8 \%$ respectively, with comparative amiodarone death rates of $21 \%$ and $41 \%$. Adjusted all cause five year mortality for ICD treatment was estimated to be $35.8 \%$ (this being $4.4+((21.8$ $+41 \cdot 0) / 2)$ ) and $52.4 \%$ for amiodarone.

In table 3 life expectancy with ICD is estimated for the adjusted mortality series to be 11.1 years and 6.7 years with amiodarone. The difference in life expectancies discounted at $6 \%$ is 2.3 years in favour of ICD. Overall, in the 20 year period of the projected costs and benefits of the study, a range of 1.7 to 3.7 discounted life years would be gained from ICD treatment.

Table 4 shows total cost and cost effectiveness data. For the adjusted mortality series the total discounted cost of ICD treatment over 20 years is $£ 28400$ compared with $£ 2300$ for amio-

Table 2 Mortality data for ICD and amiodarone treatment

\begin{tabular}{|c|c|c|c|c|c|}
\hline \multirow[b]{2}{*}{$\begin{array}{l}\text { Follow up } \\
\text { year }\end{array}$} & \multicolumn{5}{|c|}{ ICD treatment (Winkle et al, ${ }^{8} n=270$ )/amiodarone treatment (Herre et al, ${ }^{7} n=462$ ) } \\
\hline & $\begin{array}{l}\text { \% Sudden } \\
\text { deaths }\end{array}$ & $\begin{array}{l}\% \text { Non-sudden } \\
\text { deaths }\end{array}$ & $\begin{array}{l}\% \text { All cause } \\
\text { deaths }\end{array}$ & $\begin{array}{l}\text { Mean } \\
\text { \% Non-sudden } \\
\text { deaths }\end{array}$ & $\begin{array}{l}\% \text { Adjusted } \\
\text { all cause } \\
\text { deaths }\end{array}$ \\
\hline $\begin{array}{l}1 \\
3 \\
5\end{array}$ & $\begin{array}{l}0.9 / 9.0 \\
4.4 / 15.0 \\
4.4 / 21.0\end{array}$ & $\begin{array}{r}6 \cdot 8 / 15 \cdot 0 \\
14 \cdot 0 / 28 \cdot 0 \\
21 \cdot 8 / 41 \cdot 0\end{array}$ & $\begin{array}{r}7 \cdot 7 / 24 \cdot 0 \\
18 \cdot 4 / 43 \cdot 0 \\
26 \cdot 2 / 62 \cdot 0\end{array}$ & $\begin{array}{l}10 \cdot 9 \\
21 \cdot 0 \\
31 \cdot 4\end{array}$ & $\begin{array}{l}11 \cdot 8 / 19 \cdot 9 \\
25 \cdot 4 / 36 \cdot 0 \\
35 \cdot 8 / 52 \cdot 4\end{array}$ \\
\hline
\end{tabular}

«The mean non-sudden death rate for the two groups combined at each follow up time is added to the sudden death rate to derive the adjusted all cause death rate. 
Table 3 Declining exponential approximation of life expectancy and discounted life-years gained from ICD treatment

\begin{tabular}{|c|c|c|c|c|}
\hline & \multicolumn{2}{|c|}{ Adjusted mortality } & \multicolumn{2}{|c|}{ Unadjusted mortality } \\
\hline & $I C D$ & Amiodarone & $I C D$ & Amiodarone \\
\hline $\begin{array}{l}\text { Mean annual mortality rate }(\mathrm{m}) \\
\text { Life expectancy }(\mathrm{l} / \mathrm{m}) \\
\text { Life expectancy discounted at } 6 \% \text { per year } \\
\text { Total discounted life-years gained from ICD } \\
\text { Discounted life-years gained from ICD in } 20 \text { years }\end{array}$ & $\begin{array}{l}0.09 \\
11 \cdot 10 \\
7 \cdot 9 \\
2 \cdot 3 \\
1 \cdot 7\end{array}$ & $\begin{array}{l}0 \cdot 15 \\
6 \cdot 67 \\
5 \cdot 6 \\
-\end{array}$ & $\begin{array}{c}0.06 \\
16.60 \\
10.1 \\
5.9 \\
3.7\end{array}$ & $\begin{array}{l}0 \cdot 19 \\
5 \cdot 30 \\
4 \cdot 2 \\
-\end{array}$ \\
\hline
\end{tabular}

For the adjusted ICD mortality series the cumulative proportion surviving, $S(t)$, to $t=5$ years $(0.64$ from table 2$)$ is determined by $S(t)=\exp (-m t)$ where $m=0.09$ and $t=5$.

darone, giving a net cost of $£ 26100$ for ICD treatment. Taking the ratio of the net cost of ICD treatment to the net benefit ( $1 \cdot 7$ discounted life-years) gives the cost effectiveness of ICD therapy as $£ 15400$ per life-year gained. The same calculations for the unadjusted survival series show a much lower cost effectiveness ratio of $£ 8200$ per life-year gained.

The sensitivity analysis in table 5 shows that cost effectiveness calculations are most sensitive to alternative estimates of patient survival, being $£ 15400$ per life year gained for the mortality series adjusted for between study differences in non-sudden death rates and $£ 8200$ for the unadjusted mortality data. Analysis by alternative treatment assumptions for each mortality series suggests that reductions in the fixed "start up" costs of treatment due to initial ICD implantation will generate a modest improvement in cost effectiveness ( $£ 14590$ per life-year, adjusted series) when compared with reductions in recurrent cost due to factors such as increased life expectancy of the device from four to six years $(£ 12297$ per life-year).

\section{Discussion}

In a recent editorial Campbell concluded that the implantable defibrillator is an effective treatment for those at risk of sudden cardiac death and that cost is the main constraint upon the wider use of this treatment in the United Kingdom. ${ }^{14}$ Cost effectiveness analysis offers a standardised framework for assessing and combining costs and effects to permit comparison

Table 4 Costs, effects, and cost-effectiveness of ICD treatment

\begin{tabular}{lll}
\hline & Adjusted mortality & Unadjusted mortality \\
\hline Total discounted cost to 20 years: & & \\
ICD treatment & $£ 28400$ & $£ 32300$ \\
Amiodarone treatment & $£ 2300$ & $£ 2100$ \\
Net cost of ICD treatment & $£ 26100$ & $£ 30200$ \\
Net effectiveness of ICD & 1.7 life-years & 3.7 life-years \\
Net cost effectiveness of ICD & $£ 15400$ per LYG & $£ 8200$ per LYG \\
\hline
\end{tabular}

Cost effectiveness is calculated as the ratio of net cost to net effectiveness for each mortality series. LYG, life-years gained.

Table 5 Sensitivity analysis of cost effectiveness estimates

\begin{tabular}{lll}
\hline & \multicolumn{2}{l}{ Cost per life-year gained $(£)$} \\
\cline { 2 - 4 } \cline { 2 - 3 } & $\begin{array}{l}\text { Adjusted } \\
\text { mortality }\end{array}$ & $\begin{array}{c}\text { Unadjusted } \\
\text { mortality }\end{array}$ \\
\hline Base estimate & 15400 & 8200 \\
Cost of ICD implantation surgery reduced by $50 \%$ to $£ 1500$ & 14590 & 7769 \\
Device generator price reduced by 20\% to $£ 7600$ & 13029 & 6890 \\
Average generator life increased from 4 to 6 years & 12297 & 6384 \\
Follow up frequency reduced from 3 monthly to 6 monthly & 14776 & 7784 \\
Amiodarone management costs increased by $£ 1000$ per year & 12954 & 7221 \\
\hline
\end{tabular}

between new and existing treatments competing for limited resources.

Our study shows that, compared with management with amiodarone, the cost effectiveness of ICD treatment lies in the range $£ 8200$ to $£ 15400$ per life-year gained. How should such cost effectiveness data be interpreted? A common and illuminating approach is to compare the results from this study with other cardiac treatment interventions to find out whether the cost per unit outcome from ICD treatment is greater or less than other treatments that are funded through the NHS. Whereas some cardiac interventions such as heart transplantation generate life-years at lower cost than ICDs ( $£ 5000$ per quality adjusted life year, 1985 prices) some interventions are nearly equivalent or worse than ICD treatment, such as coronary bypass grafting for moderate angina with one vessel disease $(£ 12000 \text { per quality adjusted life-year })^{15}$ and drug treatment (in addition to diet) for lowering blood cholesterol to reduce coronary mortality ( $£ 19000$ per quality adjusted life year). ${ }^{16}$

Sensitivity analysis indicates that the main source of variation in estimates of cost effectiveness relates to uncertainty about the size of the mortality benefit attributable to ICD treatment. In our study this uncertainty is due, in part, to questions of comparability of the ICD and amiodarone series and the extent to which the mortality data can be generalised to other patient groups. For example, recent smaller series have reported somewhat higher survival with amiodarone, ${ }^{17} 18$ and with ICD. ${ }^{19}$ Economic evaluation ultimately depends on the strength of the epidemiological evidence on which it is based, and for this reason more precise estimates of size of the effects of ICD treatment from randomised controlled trials, or (at least) well organised registries, ${ }^{20}$ would be valuable.

Present estimates of cost should be viewed in the context of the rapid technological innovation surrounding the ICD device and its implantation. Such problems are commonplace in assessments of new technologies. ${ }^{21}$ At least, evaluation studies should be designed to recognise the dynamic nature of the technology and attempt to quantify the impact of possible future trends in a sensitivity analysis. In the specific case of ICDs there are three reasons why costs of ICD treatment are likely to fall in the future:

(a) The next generation of ICD devices are expected to have much longer lives (possibly up to nine years) and have capacitors that reform automatically thus reducing the need for 
frequent maintenance or device replacement; (b) Advances in methods for implanting defibrillators may result in current surgical techniques of lateral thoracotomy and median sternotomy being superseded by less invasive transvenous methods with an expectation of lower costs and risks;

(c) More widespread use of the devices and variation in device types may serve to promote price competition between manufacturers.

The validity of the estimates presented in our study is difficult to determine. One approach is to examine the extent to which our estimates are consistent or convergent with other studies. In a recent study in the United States Kupperman et $a^{22}$ estimated a cost effectiveness range for ICD versus drug treatment of US $\$ 15600$ to $\$ 29600$ per life-year gained, deriving cost data primarily from 1984 Medicare claims and survival estimates from publications before the major reports used here. An Australian technology assessment report on ICDs has also appeared and ICD cost effectiveness is estimated to range from AU\$17850 to AU $\$ 35800$ per life year, where ICD is assumed to be used only after electrophysiological testing has failed to find an effective antiarrhythmic agent. ${ }^{23}$ Results from our study are therefore consistent with other published studies and very similar in estimates of cost effectiveness.

A limitation of existing economic appraisals is the exclusion of effects that antiarrhythmic treatments may have upon patients' health related quality of life. To measure treatment benefits only in terms of life extension is to bias comparison against those treatments that have higher mortality but may maintain or improve quality of life, either through the relief of symptoms or the avoidance of adverse effects. Some follow up studies on ICD patients have explored physical and psychological effects but no prospective controlled comparative studies have yet been published. ${ }^{24-26}$ Quality of life data, however, are being collected as part of the Canadian implantable defibrillator study ${ }^{6}$ (a randomised controlled trial of ICD versus amiodarone) with a variety of measures including the Nottingham Health Profile ${ }^{27}$ and the Mental Health Inventory ${ }^{28}$ to assess psychological wellbeing.

We are grateful to Mark Anderson, John Camm, Stuart Connolly, Helmut Klein, Ronald Campbell, Anthony Nathan, and Sanjeev Saksena for their advice and comments. This paper is revised version of a report commissioned and funded by the United Kingdom Department of Health. The views expressed are those of the authors alone.

1 Mirowski M, Reid PR, Mower MM, et al. Termination of malignant ventricular arryhthmias with an implanted automatic defibrillator in human beings. $N$ Engl J Med
1980;303:322-4.

2 Brooks R, McGovern BA, Garan H, Ruskin JN. Current treatment of patients surviving out-of-hospital cardiac arrest. JAMA 1991;265:762-8.

3 Griffith MJ, Varma N, Sowton GE, et al. Use of automatic implantable defibrillators in the United Kingdom. $B$ Heart J 1990;64:101

4 Fogoros RN, Fielder SB, Elson JJ. The automatic implantable defibrillator in drug-refractory ventricular tachyarrhythmias. Ann Intern Med 1987;107:635-41.

5 Newman D, Herre J, Suave MJ, et al. The automatic implantable defibrillator and patient survival: a casecontrol study. J Am Coll Cardiol 1989;13:65a.

6 Protocol for the Canadian Implantable Defibrillator Study: Medical Research Council of Canada Grant No MA 10823 .

7 Herre JM, Sauve MJ, Malone P, et al. Long-term results of amiodarone therapy with recurrent sustained ventricular tachycardia or ventricular fibrillation. J Am Coll Cardiol 1989;13:422-9.

8 Winkle RA, Mead H, Ruder M, et al. Long term outcome with the automatic implantable cardioverter-defibrillator. J Am Coll Cardiol 1989;13:1353-61.

9 Beck JR, Kassirer JP, Pauker SG. A convenient approximation of life expectancy (The DEALE). I validation of mation of life expectancy (The DEALE). I validation of the method, and II use in medic

10 Vermeer F, Simoons ML, De Zwaan C, et al. Cost-benefit analysis of early thrombolytic treatment with intracoronary streptokinase. $\mathrm{Br}$ Heart $J$ 1988;59:527-34

11 Buxton MJ, Acheson R, Caine N, Gibson S, O'Brien BJ. Costs and benefits of the heart transplant programmes at Harefield and Papworth hospitals. London: Her Majesty's Stationery Office, 1985:34-51.

12 Price Waterhouse. Cardiothoracic Costing Study. Sheffield: Trent Regional Health Authority, 1988.

13 Keeler EB, Cretin S. Discounting of life-saving and other non-monetary effects. Management Science 1983;29:300-6. 4 Campbell RWF. Life at a price: the implantable defibrillator. Br Heart J 1990;64:171-3.

15 Williams A. The economics of coronary artery bypass grafting. $B M J 1985 ; 289: 326-9$.

16 Standing Medical Advisory Committee. Blood cholesterol testing: report to the Secretary of State for Health. London: Department of Health.

17 Myers M, Peter T, Weiss D, et al. Benefit and risks of longterm amiodarone therapy for sustained ventricular tachycardia/fibrillation: minimum of three-year follow up tachycardia/fibrillation: minimum of three-yea
in 145 patients. Am Heart $J 1990 ; 119: 8-14$.

18 Strasberg B, Kusneic J, Zlotikamien B, Mager A, Sclarovsky $\mathrm{S}$. Long-term follow-up of postmyocardial infarction patients with ventricular tachycardia or ventricular patients with ventricular tachycardia or ventricular
fibrillation treated with amiodarone. $\mathrm{Am} J$ Cardiol 1990;66:673-8.

19 Fogoros RN, Elson JJ, Bonnet CA, Fiedler SB, Burkholder JA. Efficacy of the automatic implantable cardioverter defibrillator in prolonging survival in patients with severe underlying cardiac disease. J Am Coll Cardiol 1990; 16:381-6.

20 Furman $S$. Implantable cardioverter defibrillator statistics. PACE 1990;13:1-2.

21 Feeny D, Guyatt G, Tugwell P. Health Care Technology: Effectiveness, efficiency and public policy. Montreal: Institute for Research on Public Policy, 1986.

22 Kupperman M, Luce BR, McGovern B, Podrid PJ, Bigger $T$, Ruskin JN. An analysis of the cost-effectiveness of the implantable defibrillator. Circulation 1990;81:91-100.

23 Cowley DE, Conway L, Hailey D. Implantable Cardiac Defibrillators. Canberra: Australia Institute of Health: Health Care Technology Series No 5, AGPS, 1990.

24 Cooper D, Luceri R, Thurer R, Myerburg RJ. The impact of the automatic implantable defibrillator on quality of life. Clinical Progress in Electrophysiology and Pacing 1986;
4:306-9.

5 Vlay SC, Olson LC, Frichionne GL, Friedman R. Anxiety and anger in patients with ventricular tachyarrhythmias. Responses after automatic internal cardioverter defibrillator implantation. PACE 1989;12:366-72.

26 Tchou PJ, Piasecki E, Gutmann M, Jazayeri $M$, Axtell $K$, Akhtar M. Psychological support and psychiatric Akhtar M. Psychological support and psychiatric management of patients with automatic implantable
cardioverter defibrillator. Int J Psychiatry Med cardioverter defibi

27 Hunt SM, McEwen J, McKenna SP. Measuring health status: a new tool for clinicans and epidemiologists. $J R$ Coll Gen Pract 1985;35:185-8.

28 Veit CT, Ware JE Jr. The structure of psychological distress and well-being in general populations. J Consult Clin Psychol 1983;51:730-42. 